\title{
Hypoxia-Ischemia Stimulates Hippocampal Glutamate Efflux in Perinatal Rat Brain: An In Vivo Microdialysis Study ${ }^{1}$
}

\author{
FAYE S. SILVERSTEIN, BINA NAIK, AND JENNIFER SIMPSON
}

Departments of Pediatrics and Neurology, University of Michigan, Ann Arbor, Michigan 48109-0570

\begin{abstract}
We used in vivo microdialysis to determine the impact of a focal hypoxic-ischemic insult on hippocampal amino acid efflux in the immature brain. Microdialysis probes were inserted into the right hippocampus of postnatal $\mathbf{d} 7$ rats. To induce hypoxic-ischemic injury, the right carotid artery was ligated and animals were exposed to $8 \%$ oxygen for $2 \mathrm{~h}$ ( $n=6$, histologically verified). Ten 20 -min dialysis fractions were collected from each animal: three sequential 20-min baseline samples, six samples during hypoxia, and a recovery sample in room air. Eight amino acids were detected in dialysates with a HPLC assay. There was marked intra- and interanimal variation in glutamate efflux; mean glutamate efflux increased from $17 \mathrm{pmol} / \mathrm{min}$ in baseline samples to 51 in the $2 \mathrm{nd} h$ of hypoxia ( $p=$ $\mathbf{0 . 0 0 2}$, Kruskal Wallis test). There was a concurrent decline in glutamine efflux (310 to $207 \mathrm{pmol} / \mathrm{min}, p=0.0005)$. Alanine efflux doubled during hypoxia $(p=0.015)$. There were no changes in efflux of the other five amino acids analyzed. In this experimental model of perinatal stroke, during the acute evolution of hypoxic-ischemic brain injury, transient large increases in glutamate efflux and corresponding declines in glutamine efflux were detected. These data support the hypothesis that synaptic concentrations of the endogenous excitatory amino acid glutamate increase during the evolution of hippocampal ischemic injury. ( $\mathrm{Pe}$ diatr Res 30: 587-590, 1991)
\end{abstract}

\section{Abbreviations}

EAA, excitatory amino acids PND, postnatal day ECF, extracellular fluid

Considerable experimental evidence indicates that endogenous EAA contribute to the pathogenesis of ischemic hippocampal injury (1-4). In perinatal brain, the immaturity of hippocampal synaptic circuitry might suggest that the contribution of endogenous EAA to the evolution of ischemic neuronal injury would be reduced, as compared with the adult. However, because PND 7 rats are susceptible to excitotoxic neuronal damage $(5,6)$, elevated synaptic concentrations of endogenous glutamate or aspartate could be neurotoxic at this developmental stage. In a perinatal rodent stroke model, i.e. stroke induced by right carotid artery ligation followed by exposure to moderate hypoxia in

Received April 22, 1991; accepted July 18, 1991

Correspondence and reprint requests: Dr. F. S. Silverstein, Room 6028 , Kresge II Building, Box 0570, University of Michigan, Ann Arbor, MI 48109-0570.

Supported by USPHS Grants NS01171 and 26142 (to F.S.S.) and Neuroscience Training Grant 2T32-NS 07222 (to J.S.).

'A preliminary report of these data was presented at the annual meeting of the Society for Pediatric Research, New Orleans, LA, May 1991.
PND 7 rats (7), considerable data suggest that endogenous glutamate plays an important role in the pathogenesis of brain injury; for example, striatal ECF glutamate content peaks transiently during the evolution of injury (8), and systemically administered glutamate antagonists attenuate the severity of tissue damage (9-11).

The hippocampus is particularly susceptible to ischemic neuronal injury. In this perinatal model, the propensity for selective CA1 pyramidal cell damage that characterizes adult ischemia models (12) is not observed (6), and the distribution of hippocampal histopathology is more variable.

In this study we used in vivo microdialysis to examine hippocampal amino acid efflux during the evolution of hypoxicischemic injury. With in vivo microdialysis, sequential changes in ECF content of amino acids can be readily estimated within defined brain regions $(13,14)$. ECF accumulation of neurotransmitter amino acid reflects regional synaptic concentrations; increased concentrations of EAA could result from increased release [from neurotransmitter or metabolic pools $(1-4,15,16)]$ or suppression of neuronal and glial reuptake $(17,18)$. Calculated efflux rates provide estimates of regional ECF content of compounds of interest.

We measured hippocampal amino acid efflux to determine if EAA efflux increased during the evolution of a focal hypoxicischemic injury in the immature brain.

\section{MATERIALS AND METHODS}

Microdialysis probes were implanted into hippocampi of PND 7 Sprague-Dawley rats, using a modification of our previously described methods for striatal dialysis $(19,20)$. Probes had a concentric design a and 2-mm dialyzing tip (diameter $250 \mu \mathrm{m}$, molecular weight cutoff 6000; Spectra-Por, Spectrum Medical, Los Angeles, CA). In preliminary experiments, we attempted to optimize stereotaxic coordinates for probe insertion. The site of probe placement was verified by postmortem examination of sequential horizontal brain sections and was considered acceptable if a discrete (probe-induced) anatomical defect was identified within the right hippocampus.

Coordinates used to guide probe insertion were (referenced to bregma): $3.5 \mathrm{~mm}$ lateral, $3.5 \mathrm{~mm}$ posterior, and $3 \mathrm{~mm}$ depth. Although these coordinates were accurate for intrahippocampal stereotaxic injections, they were not as reliable for sustained probe placement; the overall success rate for completion of sampling with verification of probe placement within hippocampus was $<50 \%$. Figure 1 demonstrates the most common site of probe placement within midhippocampus, adjacent to the superior blade of the dentate gyrus; however, using the same coordinates, probe placement sites were also identified more medially, adjacent to the CA1 pyramidal cell region and more laterally adjacent to CA3. The curvature of the skull surface made stabilization of the probe more difficult than for striatal insertions, and in about one fourth of the animals mechanical problems 


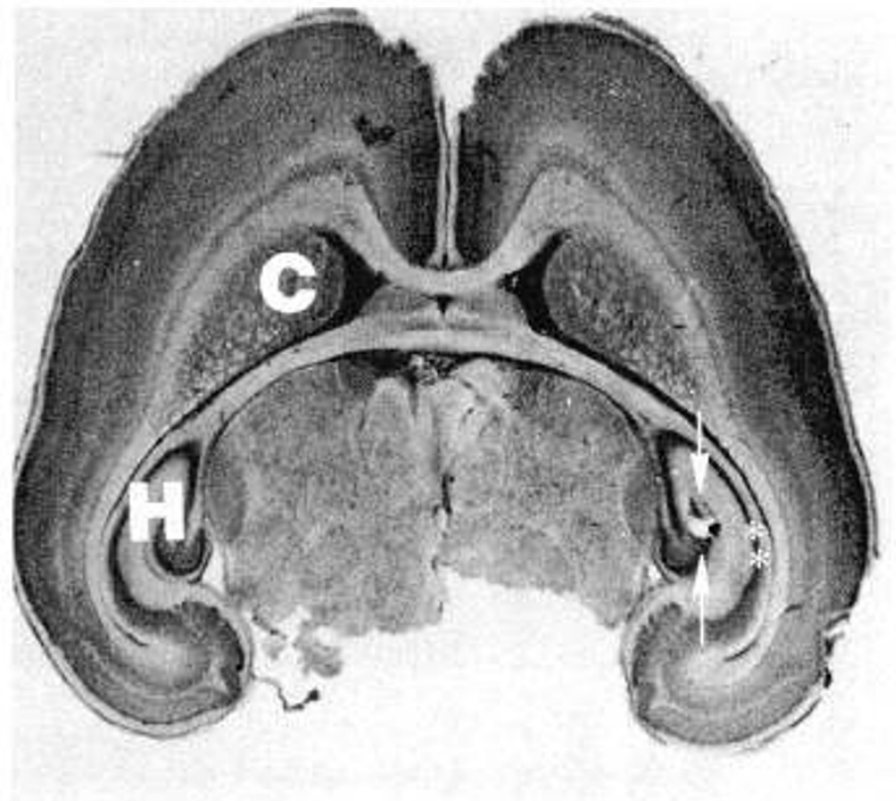

Fig. 1. Photograph of a cresyl violet-stained 50- $\mu \mathrm{m}$ horizontal brain section prepared from an unlesioned PND 7 rat that underwent in vivo hippocampal microdialysis. The anatomical defect resulting from probe insertion is evident in the superior blade of the dentate gyrus (arrowheads) of the right hippocampus. $\mathrm{C}$, caudate; $\mathrm{H}$, hippocampus; ${ }^{* *}, \mathrm{CA} 1$ pyramidal cell layer.

prevented completion of experiments. Based on these factors and on our considerable striatal microdialysis data that demonstrated stable amino acid efflux over up to $4 \mathrm{~h}$, we limited analysis to hypoxic-ischemic animals.

In ether-anesthetized animals, a midline scalp flap was excised and the right carotid artery ligated in about $5 \mathrm{~min}$. Two h later, pups were reanesthetized and probes were inserted. Probes were perfused with filtered modified Ringer's solution $(\mathrm{NaCl} 147 \mathrm{mM}$, $\mathrm{KCl} 4 \mathrm{mM}, \mathrm{CaCl}_{2} 3.4 \mathrm{mM}, \mathrm{pH} 7.2$ ) at $1.5 \mu \mathrm{L} / \mathrm{min}$ using a microinfusion pump (CMA 100, BAS). Sampling was initiated after a 2 -h recovery period, and 10 sequential 20 -min fractions were collected (on ice)

After three baseline samples were collected, animals were exposed to $8 \%$ oxygen $/ 92 \%$ nitrogen for $2 \mathrm{~h}$; then, for the final sample, animals were returned to room air. Surface body temperature was monitored and was maintained at about $35.5^{\circ} \mathrm{C}$. Animals were killed by decapitation at the end of sampling, and normoglycemia was verified (by Dextrostix $\geq 80$ ) of blood welled at the severed neck vessels. The microdialysis protocol was approved by the University of Michigan Committee on Use and Care of Animals.

Samples were analyzed by HPLC with electrochemical detection, and precolumn derivitization (modified from Ref. 21). Each sample $(30 \mu \mathrm{L})$ was mixed with $22.5 \mu \mathrm{L}$ of a solution containing $27 \mathrm{mg} 0$-pthaldialdehyde in $2 \mathrm{~mL}$ of $100 \mathrm{mM}$ borax with $20 \mu \mathrm{L}$ mercaptoethanol, $\mathrm{pH} 9.5$, for $30 \mathrm{~s}$. The internal standard homoserine $(22.5 \mu \mathrm{L})$ was included. Samples $(70 \mu \mathrm{L})$ were injected onto a $\mathrm{C} 18$ reverse phase column ( $5 \mu \mathrm{m}$ ODS); the mobile phase was a $0.1 \mathrm{M} \mathrm{NaH}_{2} \mathrm{PO}_{4}$, pH 6.5 buffer with $32 \%$ methanol. A glassy carbon electrode connected to an electrochemical detector (Bioanalytical Systems) was used (sensitivity $20 \mathrm{nA}$, electrode potential $0.7 \mathrm{~V}$ versus $\mathrm{Ag} / \mathrm{AgCl}$ to optimize the number of quantifiable peaks and minimize baseline noise). Values were quantified from peak heights, compared with peak heights of external standards assayed concurrently, and normalized using the internal standard homoserine. Values were also corrected for the recovery efficiency for each compound (recovery range 10$20 \%$ ).

Efflux values were expressed both in $\mathrm{pmol} / \mathrm{min}$ and as a percentage of baseline; means ( \pm SEM) for each sampling period were calculated. Values were also grouped for analysis into four time periods: 1) baseline, 2) $1 \mathrm{st} \mathrm{h}$ of hypoxia, 3) $2 \mathrm{nd} \mathrm{h}$ of hypoxia, and 4) recovery. Because variances were not equal, nonparametric tests were used for statistical analysis with a microcomputer-based statistical package (Statview).

\section{RESULTS}

Glutamate, aspartate, glutamine, glycine, asparagine, taurine, serine, and alanine were detected in hippocampal dialysate samples, as were several unidentified peaks; $\gamma$-aminobutyric acid was not consistently detected (despite an assay threshold of 2-3 pmol/ $20 \mu \mathrm{L}$ sample). There was considerable inter- and intraanimal variability in efflux values. Baseline efflux values were in a range similar to those obtained in striatum, using the same methods (Table 1) (20); hippocampal glutamate, aspartate, and taurine efflux values were higher than corresponding striatal values. Of interest, baseline hippocampal glutamate efflux was higher than peak values detected in striatum during hypoxia-ischemia (17 versus $7 \mathrm{pmol} / \mathrm{min}$ ); efflux values appear to estimate relative changes in ECF amino acid content more accurately than their absolute synaptic concentrations.

Preliminary analysis indicated that glutamate efflux peaked transiently and variably in the $2 \mathrm{nd} h$ of hypoxia. Because previous studies in this model suggested that the time threshold for onset of irreversible injury occurs during the $2 \mathrm{nd} h$ of hypoxic exposure (22), values from the 1 st $h$ of hypoxia (fractions 4-6) and from the 2 nd h of hypoxia (fractions 7-9) were grouped for analysis.

Figure 2 compares the widely divergent efflux patterns of the eight amino acids analyzed over these four time periods. Glutamate efflux increased during hypoxic exposure; in the $2 \mathrm{nd} \mathrm{h}$ of hypoxia, mean values were three times as high as at baseline (51 \pm 9 versus $17 \pm 3 \mathrm{pmol} / \mathrm{min}, p=0.002$, Kruskal Wallis test comparing all values in each time period), and values declined toward baseline in the final recovery period in room air. In contrast, glutamine efflux declined progressively to $60 \%$ of baseline in the final sample (311 to $182 \mathrm{pmol} / \mathrm{min}, p=0.0005$, Kruskal Wallis test). Hypoxic exposure stimulated a sustained increase in alanine efflux (from $31 \mathrm{pmol} / \mathrm{min}$ at baseline to 63 $\mathrm{pmol} / \mathrm{min}$ in the $1 \mathrm{st} \mathrm{h}$ of hypoxia). No significant changes in efflux of the five other amino acids analyzed were detected.

To facilitate more detailed comparison of sequential changes in glutamate and glutamine efflux, for each animal values were normalized and expressed as a percentage of baseline (averaged from first three fractions) (Fig. 3). Mean glutamate efflux peaked early in the 2 nd h of hypoxia, whereas glutamine efflux declined progressively.

Table 1. Comparison of baseline hippocampal and striatal amino acid efflux in PND 7 rats*

\begin{tabular}{lcc}
\hline & \multicolumn{2}{c}{ Efflux values (pmol/min) } \\
\cline { 2 - 3 } Amino acid & Hippocampus & Striatum \\
\hline Glutamate & $17.3 \pm 3.1$ & $2.1 \pm 0.5$ \\
Aspartate & $6.2 \pm 1.2$ & $1.3 \pm 0.5$ \\
Taurine & $592 \pm 125$ & $13.3 \pm 0.2$ \\
Asparagine & $2.6 \pm 0.8$ & $17.5 \pm 3.5$ \\
Glutamine & $311 \pm 20$ & $384 \pm 47$ \\
Serine & $45.4 \pm 3.4$ & $75.2 \pm 7.8$ \\
Alanine & $30.9 \pm 2.5$ & $40.2 \pm 4.5$ \\
Glycine & $44.1 \pm 5.2$ & $35.6 \pm 2.4$ \\
\hline
\end{tabular}

* Results were obtained from in vivo microdialysis experiments in PND 7 rats (see Materials and Methods). Baseline hippocampal efflux values $( \pm$ SEM) were averaged from the first three dialysis fractions collected in each animal $(n=6)$. Striatal values, obtained using the same methods, were reported previously (20). 

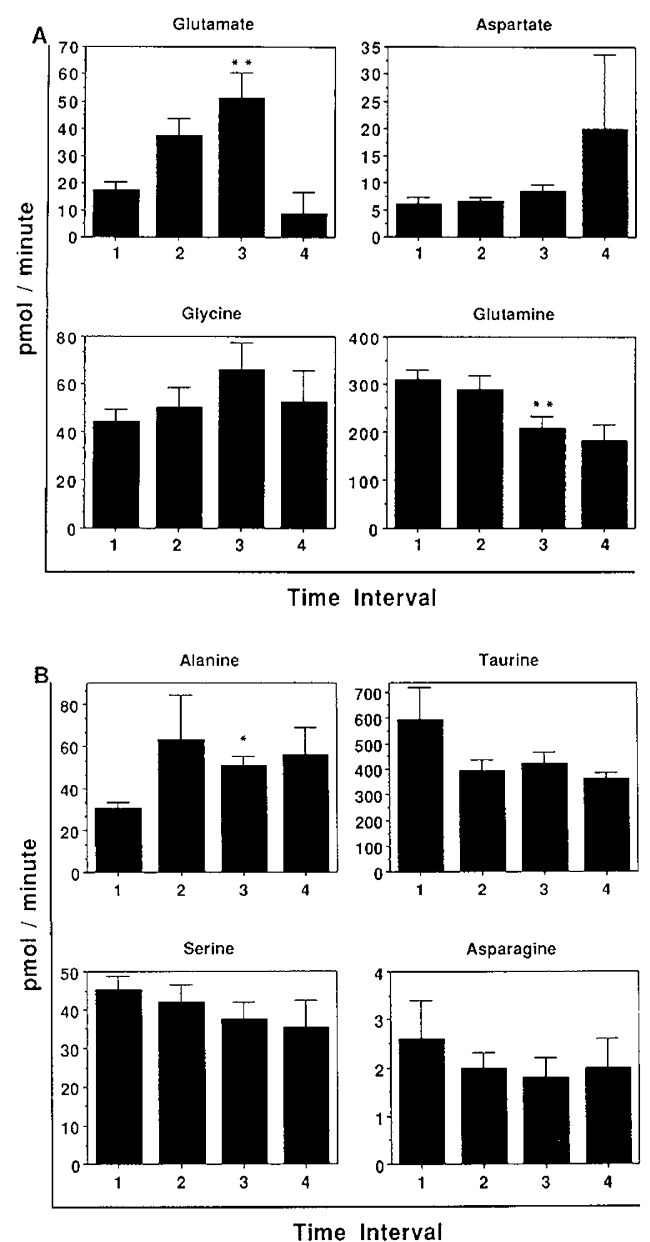

Fig. 2. $A$ and $B$. These graphs compare efflux patterns of eight amino acids detected in hippocampal dialysates of PND 7 rats during hypoxiaischemia. Microdialysis probes were inserted into the right hippocampus of PND 7 rats that underwent right carotid ligation, followed by exposure to $8 \%$ oxygen for $2 \mathrm{~h}$ ( $n=6$, see text for methods). Beginning $2 \mathrm{~h}$ after probe insertion, 10 sequential 20-min dialysis samples were collected from each animal. Samples were grouped for analysis into four time intervals: period 1, baseline, fractions 1-3; Period 2, h 1 of hypoxia, fractions 4-6; period 3, h 2 of hypoxia, fractions 7-9; and period 4, recovery, fraction 10 . Amino acid content of dialysates was quantitated by an HPLC assay. Efflux values are means \pm SEM of all measurements in each time interval. ${ }^{* *}, p<0.005 ;{ }^{*}, p<0.02$, comparison of all values within each time interval with Kruskal Wallis test.

\section{DISCUSSION}

Our results demonstrate that in this experimental model of perinatal stroke, which results in incomplete, reversible forebrain ischemia, hippocampal glutamate efflux rose markedly during the time period coinciding with the threshold for onset of irreversible injury.

Each efflux value reflects the cumulative influence of many neurons and glia over a prolonged sampling period relative to synaptic events $(13,14)$, and measurable changes in efflux indicate marked and sustained alterations in ECF glutamate accumulation. Yet, the extent of stimulation of glutamate efflux was considerably less than values reported in adult brain (3-fold compared with a $>8$-fold rise in an adult stroke model) (3). This difference may reflect lower concentrations of synaptic glutamate than in adult brain, stimulation of release (and/or suppression of reuptake) in a smaller proportion of neurons, or a greater dilutional effect by the relatively larger ECF volume in the immature brain. Differences in the pathophysiology of ischemia induction (e.g. incomplete versus complete, focal versus global),

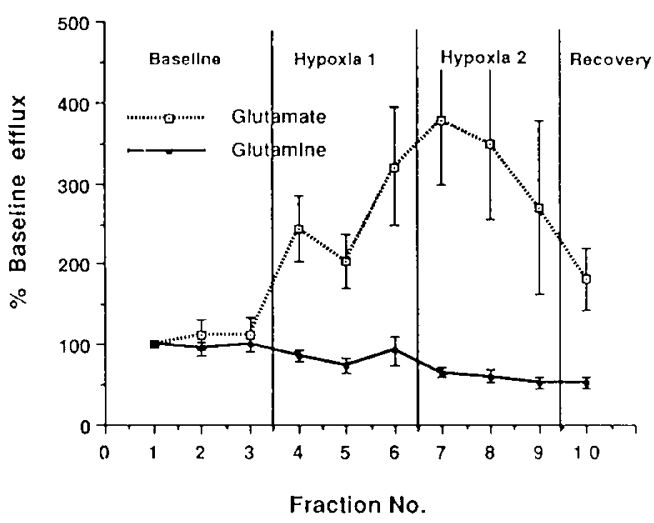

Fig. 3. this graph compares sequential hippocampal glutamate and glutamine efflux values, expressed as a percentage of baseline, in six animals that underwent right carotid artery ligation followed by $2 \mathrm{~h}$ of $8 \%$ oxygen exposure (see text for details of methods). Baseline values were defined as the averages from the first three fractions collected in each animal.

independent age-related effects, may also have contributed to the less-pronounced rise (8).

At least two major factors contributed to the wide range of efflux values observed. The extent of injury elicited by this lesioning method varies considerably; prominent lesions (with $>10 \%$ reduction in ipsilateral hemisphere mass) evolve in about $70 \%$ of animals (9), and the severity of injury in individual animals undergoing microdialysis could not be determined. There were also unavoidable differences in anatomical placement of the probe within distinct functional regions of the hippocampus, in which both baseline and stimulated release might vary. In addition, small fluctuations in brain temperature (which we were unable to control) may have influenced neurotransmitter release (23).

In contrast with glutamate, glutamine efflux declined gradually; the diverging trends for the two amino acids suggest that systemic derangements such as dehydration were unlikely to account for the increase in glutamate efflux in the $2 \mathrm{nd} h$ of hypoxia. Inhibition of the glial enzyme glutamine synthetase, which converts glutamate to glutamine, has been reported in adult brain during reperfusion after cerebral ischemia; suppression of activity of this enzyme could result in both a reduction in glutamine efflux and synaptic glutamate accumulation (24).

In adult brain, aspartate efflux generally increases in parallel with changes in glutamate efflux during ischemia $(3,4)$; however, we found no consistent increase in hippocampal aspartate efflux. Similarly, in our previous microdialysis studies in PND 7 rats, neither hypoglycemia (20) nor hypoxia-ischemia (8) stimulated striatal aspartate efflux. This difference likely reflects lower synaptic concentrations of aspartate in immature brain; whether this developmental difference in EAA metabolism has pathophysiologic significance as to mechanisms of neuronal injury is unknown. Awareness of the role of endogenous glycine in potentiating glutamate activation of $\mathrm{N}$-methyl-D-aspartate receptors has prompted interest in analysis of glycine release (25); although no significant change in glycine efflux was detected, our data revealed a trend toward increased glycine efflux in the $2 \mathrm{nd} \mathrm{h}$ of hypoxia.

Release of the endogenous potential nueromodulator taurine may increase in response to glutamatergic stimulation (26). In contrast with findings in hypoxic-ischemic striatum in which there was a close temporal correlation between glutamate and taurine efflux peaks (8), hippocampal taurine efflux did not rise. There may be regional functional differences in taurine metabolism; alternatively, because baseline hippocampal taurine efflux was much higher than in striatum, it is also possible that biologically important synaptic increases were not identifiable.

Baseline hippocampal $\gamma$-aminobutyric acid efflux was often 
undetectable, and unlike in adult brain $(3,4)$, no increases were detected with hypoxia-ischemia; immaturity of $\gamma$-aminobutyric acid innervation may also be a contributing factor to the pathophysiologic differences in the evolution of ischemic neuronal injury between immature and adult brain.

The observed increases in alanine efflux likely reflect increased glycolysis during hypoxia. Previously, in striatum, we found that both exposure to moderate hypoxia, which did not result in tissue damage, and hypoxia-ischemia stimulated alanine efflux; these findings suggested that increased alanine efflux was not an important contributing factor to the evolution of injury (8).

Our data do provide direct support of the hypothesis that extracellular glutamate accumulation may contribute to the pathogenesis of hypoxic-ischemic hippocampal injury in this model of perinatal stroke. It is also important to consider that recent studies in adult brain have demonstrated that glutamate efflux also rises during ischemia in brain regions that are relatively resistant to ischemic injury (27). Other factors, e.g. intrinsic variation in cellular susceptibility to excitotoxic injury and regional activity of endogenous neuromodulatory compounds, are likely to play critical roles in determining the extent of ischemic neuronal damage.

\section{REFERENCES}

1. Rothman S, Olney JW 1986 Glutamate and the pathophysiology of hypoxicischemic brain damage. Ann Neurol 19:105-111

2. Choi DW, Rothman SM 1990 The role of glutamate neurotoxicity in hypoxicischemic neuronal death. Annu Rev Neurosci 13:171-182

3. Benveniste H, Drejer J, Schousboe A, Diemer NH 1984 Elevation of the extracellular concentrations of glutamate and aspartate in rat hippocampus during transient cerebral ischemia monitored by intracerebral microdialysis. J Neurochem 43:1369-1373

4. Benveniste H, Jorgensen MB, Sandberg M, Christensen T, Hagberg H, Diemer NH 1989 Ischemic damage in hippocampal CA1 is dependent on glutamate release and intact innervation from CA3. J Cereb Blood Flow Metab 9:629639

5. Silverstein FS, Chen R, Johnston MV 1986 The glutamate analogue quisqualic acid is neurotoxic in striatum and hippocampus of immature rat brain. Neurosci Lett 71:13-18

6. McDonald JW, Silverstein FS, Johnston MV 1988 Neurotoxicity of NMDA is markedly enhanced in developing rat central nervous system. Brain Res 459:200-203

7. Rice JE, Vannucci RC, Brierley JB 1981 The influence of immaturity on hypoxic-ischemic brain damage in the rat. Ann Neurol 9:131-141

8. Gordon K, Simpson J, Statman D, Silverstein FS 1991 Perinatal stroke alters striatal amino acid efflux in rat brain: an in vivo microdialysis study. Stroke (in press)

9. McDonald JW, Silverstein FS, Johnston MV 1987 MK-801 protects the neonatal brain from hypoxic-ischemic damage. Eur J Pharmacol 140:359361

10. Andine $P$, Lehmann A, Ellren K, Wennberg E, Kjellmer I, Nielsen $\Upsilon$, Hagberg H 1988 The excitatory amino acid antagonist kynurenic acid administered after hypoxic-ischemia in neonatal rats offers neuroprotection. Neurosci Lett 90:209-212

11. Prince DA, Feeser HR 1988 Dextromethorphan protects against cerebral infarction in a rat model of hypoxia-ischemia. Neurosci Lett 85:291-296

12. Ito U, Sparz M, Walker JT, Klatzo I 1975 Experimental cerebral ischaemia in Mongolian gerbils. I. Light microscopic observations. Acta Neuropathol (Berl) 32:209-223

13. Ungerstedt $U 1984$ Measurement of neurotransmitter release by intracranial dialysis. In: Marsden CA (ed) Measurement of Neurotransmitter Release In Vivo. Wiley \& Sons, New York, pp 81-107

14. Benveniste H, Huttmeier PC 1990 Microdialysis-theory and application. Prog Neurobiol 35:195-215

15. Kauppinen RA, McMahon HT, Nicholls DG $1988 \mathrm{Ca}++$ dependent and $\mathrm{Ca}++$ independent glutamate release, energy status and cytosolic free $\mathrm{Ca}++$ concentration in isolated nerve terminals following metabolic inhibition; possible relevance to hypoglycemia and anoxia. Neuroscience 27:175-182

16. Nicholls D, Attwell D 1990 The release and uptake of excitatory amino acids. Trends Pharmacol Sci 11:462-468

17. Silverstein FS, Buchanan K, Johnston MV 1986 Perinatal hypoxia ischemia disrupts striatal high affinity ${ }^{3} \mathrm{H}$-glutamate uptake into synaptosomes. J Neurochem 47:1614-1619

18. Hagberg H, Lehmann A, Sandberg M, Nystrom B, Jacobson I, Hamberger A 1985 Ischemia-induced shift of inhibitory and excitatory amino acids from intra-to extracellular compartments. J Cereb Blood Flow Metab 5:413-419

19. Gordon K, Statman D, Johnston MV, Robinson TE, Becker J, Silverstein FS 1990 Transient hypoxia alters striatal catecholamine metabolism in immature brain: an in vivo microdialysis study. J Neurochem 54:605-611

20. Silverstein FS, Simpson J, Gordon KE 1990 Hypoglycemia alters striatal amino acid efflux in perinatal rats: an in vivo microdialysis study. Ann Neurol 28:515-521

21. Donzati BA, Yamamoto BK 1988 An improved and rapid HPLC-EC method for the isocratic separation of amino acid neurotransmitters from brain tissue and microdialysis perfusates. Life Sci 43:913-922

22. Silverstein FS, Johnston MV 1984 Effects of hypoxia-ischemia on monoamine metabolism in the immature brain. Ann Neurol 15:342-347

23. Minamisawa H, Nordstrom CH, Smith ML, Siesjo BR 1990 The influence of mild body and brain hypothermia on ischemic brain damage. I Cereb Blood Flow Metab 10:365-374

24. Oliver CN, Starke-Reed PE, Stadtman ER, Liu GJ, Carney JM, Floyd RA 1990 Oxidative damage to brain proteins, loss of glutamine synthetase activity, and production of free radicals during ischemia/reperfusion-induced injury to gerbil brain. Proc Natl Acad Sci USA 87:5144-5147

25. Johnson JW, Ascher P 1987 Glycine potentiates the NMDA response in cultured mouse brain neurons. Nature 325:529-531

26. Menendez N, Solis JM, Herreras O, Sanchez Herranz A, Martin del Rio R 1990 Role of endogenous taurine on the glutamate analogue-induced neurotoxicity in the rat hippocampus in vivo. J Neurochem 55:714-717

27. Globus MYT, Martinez E, Valdes K, Busto R 1990 The comparison between ischemia-induced extracellular release of neurotransmitters in vulnerable and non-vulnerable brain regions. In: Krieglstein J, Oberpichler $\mathrm{H}$ (eds) Pharmacology of Cerebral Ischemia. Verlagsgesellschaft Wissenschafftliche, Stuttgart, Germany 